\title{
Concerning the existence of Einstein and Ricci soliton metrics on solvable Lie groups
}

\author{
MiCHAEL JABLONSKI
}

\begin{abstract}
In this work we investigate solvable and nilpotent Lie groups with special metrics. The metrics of interest are left-invariant Einstein and algebraic Ricci soliton metrics. Our main result shows that one may determine the existence of a such a metric by analyzing algebraic properties of the Lie algebra and infinitesimal deformations of any initial metric.

Our second main result concerns the isometry groups of such distinguished metrics. Among the completely solvable unimodular Lie groups (this includes nilpotent groups), if the Lie group admits such a metric, we show that the isometry group of this special metric is maximal among all isometry groups of left-invariant metrics.
\end{abstract}

22E $25,53 \mathrm{C} 25,53 \mathrm{C} 30$

\section{Introduction}

Homogeneous Einstein metrics are among the simplest examples of Einstein spaces and are generally viewed as a preferred choice of metric, when they exist. Despite having been actively studied for over a century, a classification of homogeneous Einstein spaces does not seem to be close at hand.

In this work, we are primarily interested in left-invariant Einstein metrics on noncompact Lie groups. All known examples of such metrics occur on solvable Lie groups and, in fact, all known examples of noncompact homogeneous Einstein metrics are isometric to solvable Lie groups with left-invariant metrics; this is the content of the well-known Alekseevskil conjecture which has been verified in dimensions 4 and 5; see Jensen [14] and Nikonorov [32]. We refer the reader to the recent survey of Lauret [19] for a more thorough discussion of the current state of knowledge regarding Einstein metrics on Lie groups.

As there exist solvable Lie groups which cannot admit (left-invariant) Einstein metrics, we also consider the following generalization. Let $G$ be a Lie group, with Lie algebra $\mathfrak{g}$, and left-invariant metric $\langle$,$\rangle . Denote the (1,1)-$ Ricci tensor of $\{G,\langle\rangle$,$\} by Ric. By$ 
left-invariance, Ric is completely determined by its restriction to $\mathfrak{g} \simeq T_{e} G$ and we say that $\{G,\langle\rangle$,$\} is an algebraic Ricci soliton if$

$$
\operatorname{Ric}=c \operatorname{Id}+D
$$

for some $c \in \mathbb{R}$ and some $D \in \operatorname{Der}(\mathfrak{g})$. Here $\operatorname{Der}(\mathfrak{g})$ denotes the algebra of derivations of $\mathfrak{g}$. Observe, when $D=0$, the metric is an Einstein metric. When the Lie group $G$ is solvable, resp. nilpotent, such metrics have been called solvsolitons, resp. nilsolitons, in the literature. If a nilpotent Lie group admits a nilsoliton, it is called an Einstein nilradical. The only known examples of left-invariant Ricci solitons on Lie groups are solvsoliton metrics.

Question Given a solvable Lie group, how can one determine if it admits an Einstein or solvsoliton metric?

Theorem 7.1 Let $G$ be a solvable Lie group with Lie algebra $\mathfrak{g}$. The existence of a left-invariant Einstein metric on $G$ can be determined by analyzing algebraic invariants of $\mathfrak{g}$ and infinitesimal deformations of any initial left-invariant metric on $G$.

See Section 7 for complete details and an algorithm which dictates which invariants and deformations are to be measured. See Theorem 6.1 for the analogous statement for nilsolitons on nilpotent groups.

Remark The existence of an Einstein metric on a solvable Lie group is now a local question. Similarly, one can formulate the question of existence of a solvsoliton in terms of local data.

On general manifolds, the existence of an Einstein or Ricci soliton metric is not a local question. It might appear at first glance that the existence of left-invariant Einstein metrics on Lie groups is a local question since the verification of Equation (1-1) uses only the inner product and Lie bracket on $\mathfrak{g}$. However, asking if a Lie group admits such a metric amounts to asking if there exists a zero of the function $\left\|\operatorname{Ric}_{g}-\operatorname{sc}(g) / n \operatorname{Id}\right\|^{2}$ on the open set of inner products on $\mathfrak{g}$. It is not clear if this is a local question for nonsolvable Lie groups; eg, there does not exist a solution when the Lie algebra is $\mathfrak{s l}_{2} \mathbb{R}$.

In the setting of compact homogeneous spaces $G / H$, the Einstein question has received a great deal of attention and there are some partial results on the existence of such metrics. However, there are not any general, local conditions that guarantee/preclude the existence of such metrics on compact homogeneous spaces; see Bohm, Wang and Ziller [4] for the current state of research. 
Our work makes heavy use of Geometric Invariant Theory, building on the strong structural results of Heber [9] and Lauret [20]. These works take the first step in reducing the problem on the solvable group to a smaller solvable group, a one dimensional extension of a nilpotent group. This smaller solvable Lie group admits an Einstein metric if and only if its nilradical admits a so-called nilsoliton metric and the underlying Lie algebra is the extension of the nilradical by a so-called pre-Einstein derivation. Reducing the problem to analyzing the nilradical is an algebra problem.

To study the nilradical we build on the work of Nikolayevsky [31]. Using a combination of measuring algebraic information and infinitesimal deformations of metrics on the nilradical, we translate the Einstein problem into a local problem. (While we could skip this analysis on the nilradical and couple our techniques directly with the work of Heber [9, Section 6], we present our results in the given framework as these methods extend directly to solvsoliton and nilsoliton metrics. See Section 6 for more details.)

Our second main result concerns the isometry groups of solvsoliton metrics. On completely solvable unimodular Lie groups, soliton metrics are the most symmetric metrics, when they exist. This class includes nilpotent Lie groups.

Theorem 4.1 Let $S$ be a completely solvable unimodular Lie group that admits a solvsoliton metric. Let $g$ be any left-invariant metric. Then there exists a left-invariant soliton metric $g^{\prime}$ such that $\operatorname{Isom}(S, g) \subset \operatorname{Isom}\left(S, g^{\prime}\right)$, as groups.

In addition to having large isometry groups, soliton metrics are useful for decomposing the automorphism group of the underlying Lie group. In Section 5, we demonstrate a convenient way to decompose the automorphism group of Einstein nilradicals using the soliton metric. A similar procedure works for solvable groups admitting soliton or distinguished metrics.

Lastly, using the techniques presented here, we reduce the classification of solvable groups admitting Einstein, or solvsoliton metrics, to the case that the underlying Lie algebra is indecomposable.

Theorem 3.4 Let $G$ be a solvable Lie group whose Lie algebra $\mathfrak{g}=\mathfrak{g}_{1}+\mathfrak{g}_{2}$ is a direct sum of ideals. Then $G$ admits a nonflat solvsoliton, resp. flat, metric if and only if both $G_{1}$ and $G_{2}$ admit nonflat solvsoliton, resp. flat, metrics.

Corollary 3.5 Let $G$ be a solvable Lie group whose Lie algebra $\mathfrak{g}=\mathfrak{g}_{1}+\mathfrak{g}_{2}$ is a direct sum of ideals. Then $G$ admits an Einstein metric if and only if both $G_{1}$ and $G_{2}$ admit Einstein metrics of the same sign. 
A similar decomposition result has appeared for nilsolitons and nilpotent Lie groups; see Nikolayevsky [31] and Jablonski [12]. To our knowledge, the above algebraic decomposition theorem is the first of its kind for homogeneous Einstein spaces. It would be interesting to know if there is a similar theorem in the compact setting.

This work is organized as follows. Section 2 discusses the space of Lie brackets, moment maps and distinguished orbits. Section 3 compares the existence of solvsoliton metrics and distinguished orbits. Section 4 discusses isometry groups of solvsolitons. Section 5 studies the automorphism group of an Einstein nilradical. Section 6 and Section 7 show the existence of nilsoliton and Einstein metrics are intrinsic to the underlying Lie algebra, respectively.

Acknowledgements We are grateful to the referee for a thorough review and many helpful comments which have improved the presentation of this work.

\section{Preliminaries}

A Riemannian Lie group is a Lie group $G$ with a choice of left-invariant metric $\langle$,$\rangle .$ This gives rise to a metric Lie algebra $\{\mathfrak{g},\langle\rangle$,$\} , where \mathfrak{g}$ is the Lie algebra of $G$ and the inner product on $\mathfrak{g}$ is the restriction of the left-invariant metric to $T_{e} G \simeq \mathfrak{g}$. Conversely, a metric Lie algebra gives a left-invariant metric on any Lie group with said Lie algebra. We are primarily interested in simply connected Lie groups.

We say that two metric Lie algebras $\left\{\mathfrak{g}_{1},\langle,\rangle_{1}\right\}$ and $\left\{\mathfrak{g}_{2},\langle,\rangle_{2}\right\}$ are isomorphic if there exists a Lie algebra isomorphism $\phi: \mathfrak{g}_{1} \rightarrow \mathfrak{g}_{2}$ such that $\langle,\rangle_{1}=\phi^{*}\langle,\rangle_{2}$. Such an isomorphism lifts to give an isometry between the simply connected Riemannian Lie groups $\left\{G_{1},\langle\rangle,\right\} \rightarrow\left\{G_{2},\langle\rangle,\right\}$. Using such isometries, we analyze Riemannian Lie groups by considering a metric Lie algebra as a collection of three objects: a vector space $\mathbb{R}^{n}$, a Lie bracket $[\cdot, \cdot]$ and an inner product $\langle\cdot, \cdot\rangle$. Given $g \in \operatorname{GL}(n, \mathbb{R})$, we may consider a different (and isomorphic) Lie bracket $g^{*}[\cdot, \cdot]=g\left[g^{-1} \cdot, g^{-1} \cdot\right]$ and the inner product $g^{*}\langle\cdot, \cdot\rangle=\left\langle g^{-1} \cdot g^{-1} \cdot\right\rangle$. The following are isomorphic metric Lie algebras

$$
\left\{\mathbb{R}^{n}, g^{*}[\cdot, \cdot],\langle\cdot, \cdot\rangle\right\} \simeq\left\{\mathbb{R}^{n},[\cdot, \cdot],\left(g^{-1}\right)^{*}\langle\cdot, \cdot\rangle\right\}
$$

via the isomorphism $g^{-1}: \mathbb{R}^{n} \rightarrow \mathbb{R}^{n}$. In this way, we convert our questions on leftinvariant metrics of a Lie group into questions on different (but isomorphic) underlying Lie structures. 


\section{The variety of Lie brackets}

Consider the vector space $V=\wedge^{2}\left(\mathbb{R}^{n}\right)^{*} \otimes \mathbb{R}^{n}$ of antisymmetric, bilinear maps $\mu: \mathbb{R}^{n} \times \mathbb{R}^{n} \rightarrow \mathbb{R}^{n}$. This vector space is endowed with a natural $\operatorname{GL}(n, \mathbb{R})$ action:

$$
\left(g^{*} \mu\right)(v, w)=g \mu\left(g^{-1} v, g^{-1} w\right)
$$

for $g \in \mathrm{GL}(n, \mathbb{R}), v, w \in \mathbb{R}^{n}$. Via differentiation, we also have an action of $\mathfrak{g l}(n, \mathbb{R})$ on $V:(X \cdot \mu)(v, w)=X \mu(v, w)-\mu(X v, w)-\mu(v, X w)$, for $X \in \mathfrak{g l}(n, \mathbb{R})$ and $v, w \in \mathbb{R}^{n}$.

The points of $V$ can be thought of as antisymmetric algebra structures on $\mathbb{R}^{n}$, and two algebra structures are isomorphic if and only if they lie in the same $\operatorname{GL}(n, \mathbb{R})-$ orbit. Any Lie bracket $[\cdot, \cdot]$ on $\mathbb{R}^{n}$ can be realized as a point in $V$ and the subset $\mathcal{V}=\{\mu \in V \mid \mu$ satisfies the Jacobi identity $\}$ of such points is a variety in $V$. We are interested in the following subsets of $\mathcal{V}$ : let $\mathcal{N}$ denote the Lie brackets which are nilpotent and $\mathcal{S}$ denote the Lie brackets which are solvable. The varieties $\mathcal{N}, \mathcal{S}$ and $\mathcal{V}$ are $\operatorname{GL}(n, \mathbb{R})$-invariant.

We will often abuse language and refer to $\mu \in \mathcal{V}$ as a Lie algebra, when we really mean the pair $\left\{\mathbb{R}^{n}, \mu\right\}$. As it will be of interest later, we point out that the stabilizers of the actions of $\operatorname{GL}(n, \mathbb{R})$ and $\mathfrak{g l}(n, \mathbb{R})$ have relevant meaning: $\operatorname{GL}(n, \mathbb{R})_{\mu}=\operatorname{Aut}(\mu)$ and $\mathfrak{g l}(n, \mathbb{R})_{\mu}=\operatorname{Der}(\mu)$.

We now fix an inner product (the usual one) $\langle$,$\rangle on \mathbb{R}^{n}$. Given a Lie bracket $\mu \in \mathcal{V}$, we will denote by $\mathfrak{s}_{\mu}$ the metric Lie algebra $\left\{\mathbb{R}^{n},[\cdot, \cdot],\langle\cdot, \cdot\rangle\right\}$; the corresponding simply connected Lie group with left-invariant metric will be denoted by $S_{\mu}$.

\section{The moment map and geometry of orbits}

To analyze the geometry of the $\mathrm{GL}(n, \mathbb{R})$-orbits in $V$, we must first choose inner products on $V$ and $\mathfrak{g l}(n, \mathbb{R})$. The (usual) inner product on $\mathbb{R}^{n}$ extends of an $O(n)$ invariant inner product $\langle$,$\rangle on the vector space V=\wedge^{2}\left(\mathbb{R}^{n}\right)^{*} \otimes \mathbb{R}^{n}$ as follows:

$$
\langle\lambda, \mu\rangle=\sum_{i<j}\left\langle\lambda\left(e_{i}, e_{j}\right), \mu\left(e_{i}, e_{j}\right)\right\rangle=\sum_{\substack{i<j \\ k}}\left\langle\lambda\left(e_{i}, e_{j}\right), e_{k}\right\rangle\left\langle\mu\left(e_{i}, e_{j}\right), e_{k}\right\rangle
$$

where $\left\{e_{i}\right\}$ denotes the standard orthonormal basis of $\mathbb{R}^{n}$. On $\mathfrak{g l}(n, \mathbb{R})$, we consider the standard inner product

$$
\langle\alpha, \beta\rangle=\operatorname{tr} \alpha \beta^{t}=\sum_{i}\left\langle\alpha e_{i}, \beta e_{i}\right\rangle
$$

where $*^{t}$ denotes transpose and tr is the trace. This inner product is $\operatorname{Ad} O(n)$-invariant. 
Let $G$ be a real algebraic, self-adjoint subgroup of $\operatorname{GL}(n, \mathbb{R})$; by self-adjoint we mean that $G$ is closed under transpose. (The group being self-adjoint implies that it is reductive and, conversely, any reductive group can be made self-adjoint relative to some inner product on $\mathbb{R}^{n}$.) The inner product on $\mathfrak{g l}(n, \mathbb{R})$ restricts to an inner product on $\mathfrak{g}=$ Lie $G$ and we may define the moment map $m_{G}: V \backslash\{0\} \rightarrow \operatorname{symm}(n) \cap \mathfrak{g}$ for the action of $G$ on $V$; here $\operatorname{symm}(n)$ denotes the symmetric $n \times n$ matrices. This function is defined implicitly by

$$
\left\langle m_{G}(v), \alpha\right\rangle=\frac{1}{\|v\|^{2}}\langle\pi(\alpha) v, v\rangle \quad \text { for } \alpha \in \mathfrak{g}, v \in V
$$

where $\pi: \mathfrak{g} \rightarrow V$ is the induced action of $\mathfrak{g}$ on $V$. Observe that $m_{G}$ is fixed under rescaling in $V$; that is, for $c \in \mathbb{R}, m(c v)=m(v)$. When the group $G$ is understood, we will simply write $m=m_{G}$. The moment map defined here for noncompact reductive groups is a natural extension of the usual one defined for compact groups; cf Eberlein and Jablonski [7].

Using the inner product on $\mathfrak{g}$, we consider the norm squared of the moment map

$$
F=\|m\|^{2}: V \backslash\{0\} \rightarrow \mathbb{R} .
$$

Notice that this function is invariant under rescaling in $V$ and so it may be viewed as a function on spheres in $V$ or on projective space $\mathbb{P} V$. The critical points of this function have been extensively studied so as to develop a good understanding of the socalled "nullcone" of complex representations; see Kirwan [16] and Ness [27]. Moment maps have also been used in the study of general representations of noncompact real reductive groups to study the geometry of orbits; see eg Richardson and Slodowy [34], Marian [23], Eberlein and Jablonski [7] and Jablonski [11].

Definition 2.1 An orbit $G \cdot v \subset V$ is called distinguished if it contains a critical point of the function $F=\|m\|^{2}$.

Observe that $v \in V$ is a critical point of $F$ if and only if $\pi(m(v))(v)=r v$ for some $r \in \mathbb{R}$. It is a fact that any closed orbit is distinguished with critical value 0 and so these orbits are a natural generalization of closed orbits [11]. The following theorem from [17] motivates a deeper study of $\|m\|^{2}$.

Theorem 2.2 (Lauret) Let $N_{\mu}$ denote the simply connected nilpotent Riemannian Lie group whose Lie algebra $\mathfrak{n}_{\mu}$ (with inner product) corresponds to the point $\mu \in \mathcal{N}$. Then $N_{\mu}$ is a nilsoliton if and only if $\mu$ is a critical point of $F(v)=\|m\|^{2}(v)$. 
Equivalently, $N_{\mu}$ is an Einstein nilradical if and only if the orbit $\operatorname{GL}(n, \mathbb{R}) \cdot \mu$ is distinguished. The above relationship between Einstein nilradicals and distinguished orbits has been studied extensively in the literature; see eg Lauret [17] Will [37; 38], Payne [33], Lauret and Will [22], Eberlein [6], Jablonski [11; 13] and Nikolayevsky [28; $29 ; 30 ; 31]$. Motivated by this, we explore the relationship between distinguished orbits and soliton metrics on solvable groups (cf Proposition 3.1).

Definition 2.3 A Riemannian Lie group $S_{\mu}$ is said to have a distinguished metric if $\mu$ is a critical point of $F=\|m\|^{2}$ for the action of $\operatorname{GL}(n, \mathbb{R})$ on $V$ (defined above).

Remark 2.4 Geometrically, the moment map can be understood as follows. When $\mu$ is a nilpotent Lie algebra, $m(\mu)=4 \operatorname{Ric}\left(N_{\mu}\right)$. More generally, if $\mu$ is any Lie algebra with corresponding Lie group $S_{\mu}$, then $m(\mu)=4 R$ where $R$ is the tensor appearing in the formula

$$
\text { Ric }=R-\frac{1}{2} B-S(\operatorname{ad} H)
$$

here Ric is the Ricci tensor of $S_{\mu}, B$ is the Killing form of the Lie algebra $\mu$ and $S(\operatorname{ad} H)=\frac{1}{2}\left(\operatorname{ad} H+\operatorname{ad} H^{t}\right)$, where ad $H$ is a mean curvature vector. See Besse [2, Corollary 7.38] for more discussion and details.

\section{Bracket flow}

As the critical points of $F=\|m\|^{2}$ are related to the existence of soliton metrics, we are motivated to analyze the behavior of the negative gradient flow of $F$.

Let $G$ be an algebraic, self-adjoint subgroup of $\operatorname{GL}(n, \mathbb{R})$ and define $K_{G}=G \cap O(n)$. Denote the moment map of this group action by $m_{G}$ and consider the function $F=$ $\left\|m_{G}\right\|^{2}$ with critical set $\mathfrak{C}^{G}$. Denote the negative gradient flow of $F$ by $\varphi_{t}$. Given a Lie algebra $\mu \in \mathcal{V}, \varphi_{t}(\mu) \subset \mathcal{V}$ and we refer to this evolution as the bracket flow of $\mu=\mu_{0}$.

Definition 2.5 The $\omega$-limit set of $\varphi_{t}(p) \subseteq V$ is the set $\left\{q \in V \mid \varphi_{t_{n}}(p) \rightarrow q\right.$ for some sequence $t_{n} \rightarrow \infty$ in $\left.\mathbb{R}\right\}$. We denote this set by $\omega(p)$.

Proposition 2.6 (Sjamaar [35]) The omega limit set $\omega(p)$ is a single point.

The uniqueness of limits is a strong result and is due to the fact that $F=\|m\|^{2}$ is real analytic. As the limit is well-defined, we will denote it by $\omega(p)=\varphi_{\infty}(p)$. We point out that many of the following results can be proven without knowing that there is a unique point in the limit set. 
Theorem 2.7 (Jablonski [12]) Consider $p \in \mathfrak{C}^{G}$. Then

(1) $F(p)$ is a minimum of $F$ restricted to $G \cdot p$,

(2) $\mathfrak{C}^{G} \cap\{$ sphere of radius $\|p\|\} \cap G \cdot p=K_{G} \cdot p$,

(3) $\omega(G \cdot p)=K_{G} \cdot p$, that is, $\varphi_{\infty}(g p) \in K_{G} \cdot p$ for all $g \in G$.

Observe that (iii) implies: if $G \cdot p$ contains a critical point of $F$, then $\varphi_{\infty}(p) \in G \cdot p$. The first two statements originally appeared in Kempf and Ness [15] for complex representations and in Marian [23] for real representations. The third statement has appeared in Jablonski [12] but can also be derived from the work of Heinzner, Schwarz and Stötzel [10].

\section{Soliton metrics and distinguished orbits}

Let $\mathfrak{g}$ be a solvable Lie algebra with nilradical $\mathfrak{n}$. We say that an inner product $\langle$,$\rangle is standard if \mathfrak{a}=\mathfrak{n}^{\perp}$ is abelian; the associated Riemannian Lie group will also be called standard. If a Riemannian Lie group $\{G,\langle\rangle$,$\} is Ricci flat, then it is$ necessarily solvable and the metric is standard; see Alekseevskil and $\mathrm{Kimel}^{\prime} \mathrm{fel}^{\prime} \mathrm{d}$ [1] and Milnor [24]. Only recently was it shown that every Einstein metric on a solvable Lie group must be standard. Moreover, every solvsoliton is standard; see Lauret [20] where other strong structural results are obtained. Using these new results, we obtain the following relationship to distinguished orbits.

Proposition 3.1 Let $S_{\mu}$ be a solvable Lie group. If $S_{\mu}$ admits a solvsoliton metric, then $\mathrm{GL}(n, \mathbb{R}) \cdot \mu$ is a distinguished orbit. Moreover, if $S_{\mu}$ is a completely solvable group, then $S_{\mu}$ admits a solvsoliton if and only if $\operatorname{GL}(n, \mathbb{R}) \cdot \mu$ is distinguished.

Proof To prove this, one consults the work Lauret [18] where complex Lie algebras are studied. All the results of that paper remain true for real Lie algebras with the Hermitian transpose replaced with the usual transpose. For a detailed proof of this fact, see Jablonski [11]. We warn the reader that the moment map defined there is a multiple of the moment map defined here. If $n$ denotes the moment map from [18] and $m$ denotes the moment map used in this work, then $n=2 m$. Our choice of moment map $m$ is consistent with Lauret's in [19;21].

Case $1 S_{\mu}$ admits a flat metric. If $\mu$ corresponds to the flat metric, then $\mu$ is also a critical point of $F=\|m\|^{2}$; see [18, Theorem 4.7].

Case $2 S_{\mu}$ admits a nonflat solvsoliton. We only prove this in the case that the nilradical of $\mathfrak{s}_{\mu}$ is nonabelian. The abelian case is similar and we leave it to the diligent reader. 
The proof of this case is just a careful comparison of [21, Theorem 4.8] with [18, Theorem 4.7]. The soliton metric and the distinguished metric differ only in their values on $\mathfrak{a} \times \mathfrak{a}$, where $\mathfrak{a}=\mathfrak{n}^{\perp}$. If the nilradical (which is a nilsoliton in either case) satisfies $\operatorname{Ric}_{\mathfrak{n}}=c \operatorname{Id}+D$, for some $D \in \operatorname{Der}(\mathfrak{n})$, and has sc $=-1 / 4$, then the solvsoliton metric on $\mathfrak{a}$ is

$$
\langle A, A\rangle=\frac{-1}{c} \operatorname{tr} S(\operatorname{ad} A)^{2}
$$

where $S(\operatorname{ad} A)$ is the symmetric part of ad $A$, while the distinguished metric on $\mathfrak{a}$ is

$$
\langle\langle A, A\rangle\rangle=\frac{1}{2} \cdot \frac{-1}{c} \operatorname{tr}\left(\operatorname{ad} A(\operatorname{ad} A)^{t}\right)
$$

In [18], $\mathfrak{a}$ is viewed as a subset of $\operatorname{Der}(\mathfrak{n})$ with $A \simeq$ ad $A$. Clearly the existence of a soliton metric implies the existence of a distinguished metric (ie, critical point of $F$ ).

We now show the converse when $\mathfrak{g}$ is completely solvable. Recall that $\mathfrak{g}$ being completely solvable means ad $X: \mathfrak{g} \rightarrow \mathfrak{g}$ has only real eigenvalues for all $X \in \mathfrak{g}$. Assuming $\mathfrak{g}$ admits a distinguished metric, the above work shows that $\mathfrak{g}$ admits a soliton precisely when $\langle A, A\rangle=(-1 / c) \operatorname{tr} S(\operatorname{ad} A)^{2}$ is nondegenerate. This quadratic form is degenerate precisely when $\operatorname{ad} A$ is skew-symmetric (relative to the nilsoliton metric on $\mathfrak{n}$ ) and this cannot happen when $\mathfrak{g}$ is completely solvable.

Furthermore, we see that ad $A$ is symmetric relative to the nilsoliton metric on $\mathfrak{n}$, for $A \in \mathfrak{a}=\mathfrak{n}^{\perp}$, (which follows quickly from Theorem 4.7 and Proposition 4.8 of [18]) and the soliton and distinguished metrics on $\mathfrak{g}$ are related as follows: $\langle\langle\rangle\rangle=,\frac{1}{2}\langle$, on $\mathfrak{a} \times \mathfrak{a}$.

Remark 3.2 There are solvable groups which admit a distinguished metric, but cannot admit a solvsoliton. For example, if $\mathfrak{n}$ is a nonabelian Einstein nilradical and $\mathfrak{a} \subset \operatorname{Der}(\mathfrak{n})$ is an abelian subalgebra of skew-symmetric endomorphisms, then $S_{\mu}$ with $\mathfrak{s} \mu=\mathfrak{a} \ltimes \mathfrak{n}$ cannot admit a solvsoliton but does admit a distinguished metric; cf [18, Theorem 4.7; 21, Theorem 4.8].

Remark 3.3 Given any initial metric, observe that one may find the soliton metric by following two consecutive curves. The first curve follows the negative gradient flow of $F=\|m\|^{2}$ to a distinguished metric (cf Theorem 2.7) and the second curve picks up by simply adjusting the metric on $\mathfrak{a}$.

Using the relationship between distinguished orbits and solvsoliton metrics, we may prove the following decomposition theorem. This theorem has appeared in the literature for nilpotent groups (see Nikolayevsky [31] and Jablonski [12]), but was not previously known for solvable groups in general. 
Theorem 3.4 Let $S_{\mu}$ be a solvable Lie group whose Lie algebra $\mathfrak{s}_{\mu}=\mathfrak{s} \mu_{1}+\mathfrak{s} \mu_{2}$ is a direct sum of ideals. Then $S_{\mu}$ admits a nonflat solvsoliton, resp. flat, metric if and only if both $S_{\mu_{1}}$ and $S_{\mu_{2}}$ admit nonflat solvsoliton, resp. flat, metrics.

Corollary 3.5 Let $S_{\mu}$ be a solvable Lie group whose Lie algebra $\mathfrak{s}_{\mu}=\mathfrak{s} \mu_{1}+\mathfrak{s}_{\mu_{2}}$ is a direct sum of ideals. Then $S_{\mu}$ admits an Einstein metric if and only if both $S_{\mu_{1}}$ and $S_{\mu_{2}}$ admit Einstein metrics of the same sign.

All known examples of Lie groups admitting Einstein (and soliton) metrics satisfy such a decomposition property (as all such examples are either compact semisimple or solvable groups). However, it is not clear if an analogous statement is true for compact homogeneous spaces admitting Einstein metrics.

Lemma 3.6 Consider $\mathfrak{s}_{\mu}=\mathfrak{s}_{\mu_{1}}+\mathfrak{s}_{\mu_{2}}$ which is a sum of ideals and write $\mu=\mu_{1}+\mu_{2}$, where $\mathfrak{s}_{\mu_{i}}=\left\{\mathbb{R}^{n_{i}}, \mu_{i}\right\}$ and $n_{i}=\operatorname{dim} \mathfrak{s} \mu_{i}$. If $\operatorname{GL}(n, \mathbb{R}) \cdot \mu$ is a distinguished orbit, then both orbits $\operatorname{GL}\left(n_{i}, \mathbb{R}\right) \cdot \mu_{i} \subset \wedge^{2}\left(\mathbb{R}^{n_{i}}\right) \otimes \mathbb{R}^{n_{i}}$ are distinguished for $i=1,2$.

This has been proven in [12, Theorem 4.5] for nilpotent groups. However, the proof there only uses the fact that the orbits are distinguished and works in this setting with no modifications.

Proof of Theorem 3.4 We prove the case that the solvsoliton is not flat. The flat case is similar and we leave it to the reader.

One direction is trivial. Recall, a nonflat solvsoliton with $\operatorname{Ric}=c \operatorname{Id}+D$ satisfies $c<0$; see [21, Proposition 4.6]. If $S_{\mu_{i}}$ admit solvsolitons satisfying $\operatorname{Ric}_{\mu_{i}}=c_{i} \operatorname{Id}+D_{i}$, then one just needs to rescale so that $c_{1}=c_{2}$. Endow $\mathfrak{s}_{\mu}$ with the product metric, ie the $\mathfrak{s}_{\mu_{i}}$ are orthogonal and the restriction to $\mathfrak{s}_{\mu_{i}}$ is the aforementioned metric. Then $\mathfrak{s} \mu$, with $\mu=\mu_{1}+\mu_{2}$, is a solvsoliton satisfying $\operatorname{Ric}_{\mu}=\operatorname{Ric}_{\mu_{1}} \oplus \operatorname{Ric}_{\mu_{2}}=c_{1} \operatorname{Id}+\left(D_{1} \oplus D_{2}\right)$.

We now show the converse. Recall that $S_{\mu}$ admitting a solvsoliton implies the orbit $\operatorname{GL}(n, \mathbb{R}) \cdot \mu$ is distinguished by Proposition 3.1. By the lemma above, we may assume that $\mu_{i}$ are distinguished points and write $\mathfrak{s}_{\mu_{i}}=\mathfrak{a}_{i} \oplus \mathfrak{n}_{i}$ where $\mathfrak{n}_{i}$ is the nilradical and $\mathfrak{a}_{i}$ is a reductive subalgebra (cf [18, Theorem 4.7]). As $\mathfrak{s} \mu_{i}$ are distinguished, the nilradicals $\mathfrak{n}_{i}$ admit nilsolitons by [18, Theorem 4.7].

Write $\mathfrak{s}_{\mu}=\mathfrak{s} \mu_{1}+\mathfrak{s} \mu_{2}=\left(\mathfrak{a}_{1}+\mathfrak{a}_{2}\right)+\left(\mathfrak{n}_{1}+\mathfrak{n}_{2}\right)$. As $\mathfrak{s}_{\mu}$ is solvable, we see that the reductive subalgebra $\mathfrak{a}=\mathfrak{a}_{1}+\mathfrak{a}_{2}$ is abelian and hence each $\mathfrak{a}_{i}$ is abelian. Furthermore, for any $A \in \mathfrak{a}$, we see that ad $A: \mathfrak{n} \rightarrow \mathfrak{n}$ has no purely imaginary eigenvalues by the observations in the proof of Proposition 3.1. The nilradicals $\mathfrak{n}_{i}$ admit soliton metrics as $\mathfrak{n}=\mathfrak{n}_{1} \oplus \mathfrak{n}_{2}$ does; see [31;12]. Putting these facts together, we see that the solvable groups $S_{\mu_{i}}$ admit solvsoliton metrics by the same observations in the proof of Proposition 3.1. 
Proof of Corollary 3.5 The proof of the corollary follows immediately from the proof of Theorem 3.4 and the fact that isomorphic distinguished points must be isometric. More precisely, isomorphic distinguished points lie in the same $O(n)$-orbit; see Theorem 2.7.

\section{Isometry groups of solvsolitons}

Due to the classification results of Heber and Lauret, it is known that not all solvable Lie groups can admit Einstein metrics. As solvsolitons are a natural generalization of Einstein metrics, and unique (up to scaling) when they exist, these have been considered a natural choice of preferred metric in the absence of an Einstein metric. Moreover, on nilpotent Lie groups, soliton metrics are measurably the closest metric to being Einstein that such a group can admit (see Lauret [19] for a detailed account of such metrics). We defend this preference with the following result.

Theorem 4.1 Let $S$ be a completely solvable unimodular group admitting a solvsoliton metric. Let $\langle$,$\rangle be any left-invariant metric. Then there exists a left-invariant$ soliton metric $\langle,\rangle^{\prime}$ such that $\operatorname{Isom}(S,\langle\rangle,) \subset \operatorname{Isom}\left(S,\langle,\rangle^{\prime}\right)$, as groups.

Corollary 4.2 Let $N$ be an Einstein nilradical. Let $\langle$,$\rangle be any left-invariant met-$ ric. Then there exists a left-invariant soliton metric $\langle,\rangle^{\prime} \operatorname{such}$ that $\operatorname{Isom}(N,\langle\rangle,) \subset$ $\operatorname{Isom}\left(N,\langle,\rangle^{\prime}\right)$, as groups.

As nilpotent groups are all completely solvable and unimodular, the corollary is an immediate consequence of the theorem.

Remark In this way, we see that these soliton metrics are the most symmetric (leftinvariant) metric that such nilpotent and solvable groups can admit.

To prove Theorem 4.1, we first recall a result of Gordon and Wilson [8, Theorem 4.3].

Theorem 4.3 (Gordon-Wilson) Let $G$ be a completely solvable unimodular Lie group with left-invariant metric $\langle$,$\rangle . The full isometry group is a semidirect product$

$$
\operatorname{Isom}(G,\langle,\rangle)=K \ltimes G
$$

where $K \subset \operatorname{Aut}(G)$ is the isotropy subgroup of $\operatorname{Isom}(G,\langle\rangle$,$) preserving the identity$ $e \in G$. Under the natural identification $\operatorname{Aut}(G) \simeq \operatorname{Aut}(\mathfrak{g})$ we have $K \simeq \operatorname{Aut}(\mathfrak{g}) \cap O(\langle\rangle$,$) ,$ where $O(\langle\rangle$,$) is the orthogonal group of the inner product \langle$,$\rangle on \mathfrak{g}$. 
For any Riemannian Lie group, the group $G \rtimes(O(\langle\rangle,) \cap \operatorname{Aut}(G))$ is a subgroup of isometries. For nonunimodular solvable groups, it is well-known that the full isometry group is significantly larger than this subgroup [8].

Lemma 4.4 Consider the action of $\operatorname{GL}(n, \mathbb{R})$ on $V$ (as in Section 2) and let $G$ be a real algebraic, self-adjoint subgroup. Let $G \cdot p$ be a distinguished orbit and denote by $\varphi_{\infty}(p)$ the limit of the gradient flow of $F=\|m\|^{2}$ starting at $p$. Then $K_{p} \subset K_{\varphi_{\infty}}(p)$, where $K=G \cap O(n)$ and $K_{q}$ is the stabilizer subgroup at $q$.

Proof This lemma is an immediate consequence of Theorem 2.7 by using the $K-$ equivariance of $m_{G}$ and the uniqueness of integral curves of the negative gradient flow of $\left\|m_{G}\right\|^{2}$. In fact, one has $K_{p} \subset K_{\varphi_{t}(p)}$ and the result follows by taking the limit.

Lemma 4.5 [13] Consider an algebraic, self-adjoint subgroup $G$ of $\operatorname{GL}(n, \mathbb{R})$ acting on $V$ (as in Section 2). Suppose $H$ is an algebraic, self-adjoint group of automorphisms of $\mu \in V$. The centralizer of $H$ in $G, Z_{G}(H)=\{g \in G \mid g h=h g$ for all $h \in H, g \in G\}$, is algebraic, self-adjoint and $G \cdot \mu$ is a distinguished orbit if and only if $Z_{G}(H) \cdot \mu$ is a distinguished orbit. Moreover, along the orbit $Z_{G}(H) \cdot \mu, m_{G}=m_{Z_{G}(H)}$.

This is an immediate consequence of [13, Theorem 4.4].

Remark In the above, there is no ambiguity as to the meaning of distinguished since $m_{G}=m_{Z_{G}(H)}$ along the subset $Z_{G}(H) \cdot \mu$. Also, the group $H$ being a group of automorphisms means precisely that $H$ is a subgroup of the stabilizer of $\operatorname{GL}(n, \mathbb{R})$ at $\mu$, and $H$ being self-adjoint automatically makes $H$ reductive.

Proof of Theorem 4.1 Recall that a completely solvable unimodular Lie group $S_{\mu}$ admits a solvsoliton metric if and only if $\operatorname{GL}(n, \mathbb{R}) \cdot \mu$ is a distinguished orbit (Proposition 3.1). To show that such metrics have maximal isometry groups, we first show that there exists a distinguished metric (ie critical point of $F=\|m\|^{2}$ ) in which the isometry group embeds and then show that this metric has the same isometry group as a particular choice of soliton metric.

By the result of Gordon-Wilson above, the isometry group of a completely solvable unimodular group $S_{\mu}$ is $\operatorname{Isom}\left(S_{\mu}\right)=S_{\mu} \rtimes(\operatorname{Aut}(\mu) \cap O(\langle\rangle$,$) . Given g \in \operatorname{GL}(n, \mathbb{R})$, $\operatorname{Aut}\left(g^{*} \mu\right)=g \operatorname{Aut}(\mu) g^{-1}$ and $O\left(\left(g^{-1}\right)^{*}\langle\rangle,\right)=g^{-1} O(\langle\rangle)$,$g , where \left(g^{-1}\right)^{*}\langle\cdot, \cdot\rangle=$ $\langle g \cdot, g \cdot\rangle$. Recall that the following metric Lie algebras are isometric (see Section 2):

$$
\left\{\mathbb{R}^{n}, g^{*} \mu,\langle\cdot, \cdot\rangle\right\} \simeq\left\{\mathbb{R}^{n}, \mu,\left(g^{-1}\right)^{*}\langle\cdot, \cdot\rangle\right\} .
$$


The corresponding Riemannian solvable Lie groups are isometric:

$$
\left\{S_{g^{*} \mu},\langle\cdot, \cdot\rangle\right\} \simeq\left\{S_{\mu},\left(g^{-1}\right)^{*}\langle\cdot, \cdot\rangle\right\} .
$$

At $e \in S_{\mu}$, the isometry group of $\left\{S_{\mu},\left(g^{-1}\right)^{*}\langle\cdot, \cdot\rangle\right\}$ has isotropy subgroup

$$
\operatorname{Aut}(\mu) \cap O\left(\left(g^{-1}\right)^{*}\langle,\rangle\right)=g^{-1}\left(\operatorname{Aut}\left(g^{*} \mu\right) \cap O(\langle,\rangle)\right) g .
$$

Step 1 Consider a Riemannian solvable group $S_{\mu}$ where $\operatorname{GL}(n, \mathbb{R}) \cdot \mu$ is a distinguished orbit. As the group $H=\operatorname{Aut}(\mu) \cap O(\langle\rangle$,$) is self-adjoint, Theorem 2.7$ and Lemma 4.5 imply the orbit $Z_{\mathrm{GL}(n, \mathbb{R})}(H) \cdot \mu$ contains the limit $\mu_{\infty}$ of the negative gradient flow of $F=\|m\|^{2}$. Let $g \in Z_{\mathrm{GL}(n, \mathbb{R})}(H)$ be such that $g \cdot \mu=\mu_{\infty}$. By Lemma 4.4, we see that

$$
\operatorname{Aut}(\mu) \cap O(\langle,\rangle)=K_{\mu} \subset K_{g \cdot \mu}=\operatorname{Aut}\left(g^{*} \mu\right) \cap O(\langle,\rangle)
$$

where $K=O(n, \mathbb{R})$. Using the fact that $g \in Z_{\mathrm{GL}(n, \mathbb{R})}(H)$, we obtain

$$
\begin{aligned}
\operatorname{Aut}(\mu) \cap O(\langle,\rangle) & =g^{-1}(\operatorname{Aut}(\mu) \cap O(\langle,\rangle)) g \\
& \subset g^{-1}\left(\operatorname{Aut}\left(g^{*} \mu\right) \cap O(\langle,\rangle)\right) g=\operatorname{Aut}(\mu) \cap O\left(\left(g^{-1}\right)^{*}\langle,\rangle\right) .
\end{aligned}
$$

As the underlying Lie group structure of $\left\{S_{\mu},\langle\rangle,\right\}$ and $\left\{S_{\mu},\left(g^{-1}\right)^{*}\langle\rangle,\right\}$ is the same,

$$
\operatorname{Isom}\left(S_{\mu},\langle,\rangle\right) \subset \operatorname{Isom}\left(S_{\mu},\left(g^{-1}\right)^{*}\langle,\rangle\right)
$$

as Lie groups.

Step 2 So far we have imbedded the isometry group of $S_{\mu}$ into the isometry group of a distinguished metric $S_{\mu^{\prime}}$ (the underlying Lie structures are the same). We finish by constructing a soliton metric with the same isometry group as $S_{\mu^{\prime}}$.

Write $\mathfrak{s}_{\mu^{\prime}}=\mathfrak{a} \ltimes \mathfrak{n}$. In the proof of Proposition 3.1 it was observed that the distinguished metric $\left\langle\langle\right.$,$\rangle on \mathfrak{s} \mu^{\prime}$ can be transformed into a solvsoliton metric $\langle$,$\rangle by simply$ rescaling the metric on $\mathfrak{a}$ by a factor of 2 . As this rescaling does not change the isotropy of the isometry groups

$$
\operatorname{Aut}(\mu) \cap O(《,\rangle)=\operatorname{Aut}(\mu) \cap O(\langle,\rangle),
$$

and the underlying Lie groups are the same, these metrics have the same isometry groups and the proof is complete.

Remark 4.6 Presently, we do not have such a theorem when the group is not completely solvable or for nonunimodular solvable Lie groups. Our techniques do allow one to embed a large portion of the isometry group of any metric into the Einstein or solvsoliton metric, however they do not allow one to embed the entire isometry group. This question will be addressed in future work. 
It should be noted that every solvsoliton is isometric to a solvable Lie group which is completely solvable [21]. This gives strong motivation that the above result should hold without the requirement of being completely solvable.

\section{Automorphisms of Einstein nilradicals}

The main result of this section is a decomposition theorem for the automorphism group of an Einstein nilradical. The reader who is primarily interested in the algorithm which determines the existence of Einstein metrics on solvable Lie groups may skip to the next section.

Given $\alpha \in \operatorname{symm} \subset \mathfrak{g l}(n, \mathbb{R})$, a symmetric matrix, we define the following groups:

$$
\begin{aligned}
G_{\alpha} & =\left\{g \in \mathrm{GL}(n, \mathbb{R}) \mid g \alpha g^{-1}=\alpha\right\} \\
U_{\alpha} & =\{g \in \mathrm{GL}(n, \mathbb{R}) \mid \exp (t \alpha) g \exp (-t \alpha) \rightarrow e \text { as } \mathrm{t} \rightarrow-\infty\} \\
P_{\alpha} & =G_{\alpha} U_{\alpha}
\end{aligned}
$$

The group $P_{\alpha}$ is the parabolic associated to $\alpha$ with unipotent radical $U_{\alpha}$ and reductive complement $G_{\alpha}$. The group $G_{\alpha}$ is the centralizer of $\alpha$ in $\operatorname{GL}(n, \mathbb{R})$ and, as $\alpha^{t}=\alpha, G_{\alpha}$ is self-adjoint with Cartan decomposition $G_{\alpha}=K_{\alpha} \exp \left(\mathfrak{p}_{\alpha}\right)$; here $K_{\alpha}=G_{\alpha} \cap O(n)$ and $\mathfrak{p}_{\alpha}=\mathfrak{g}_{\alpha} \cap \operatorname{symm}(n)$, where $\operatorname{symm}(n)$ denotes the symmetric $n \times n$ matrices.

Conjugating $\alpha$ by an element of $O(n)$, we may assume that $\alpha$ is diagonal with weakly increasing eigenvalues. Now the group $G_{\alpha}$ consists of block diagonal matrices (which commute with $\alpha$ ) while $U_{\alpha}$ is the group of lower triangular elements beneath the blocks of $G_{\alpha}$.

Proposition 5.1 Let $\mu \in \mathcal{N}$ correspond to a nilsoliton $N_{\mu}$ with Ricci tensor $\alpha=$ Ric. Then the automorphism group of $N_{\mu}$ decomposes as

$$
\operatorname{Aut}(\mu)=G^{\alpha} U^{\alpha}=K^{\alpha} \exp \left(\mathfrak{p}^{\alpha}\right) U^{\alpha}
$$

where $G^{\alpha}=G_{\alpha} \cap$ Aut, $K^{\alpha}=K_{\alpha} \cap$ Aut, $\mathfrak{p}^{\alpha}=\mathfrak{p}_{\alpha} \cap$ Der, $U^{\alpha}=U_{\alpha} \cap$ Aut.

While this decomposition is interesting in its own right, we use it to streamline the algorithms in Sections 6 and 7. Recall, $\operatorname{Der}(\mu)$ is the Lie algebra of $\operatorname{Aut}(\mu)$ and so we have an analogous decomposition of $\operatorname{Der}(\mu)$. The following is presented for later use.

Lemma 5.2 Take a nilsoliton $N_{\mu}$ with Einstein derivation $\beta$, ie, $\operatorname{Ric}=c \operatorname{Id}+\beta$. Then the derivation algebra decomposes as $\operatorname{Der}(\mu)=\mathfrak{k}^{\beta} \oplus \mathfrak{p}^{\beta} \oplus u^{\beta}$. Moreover, every element of the form

$$
\beta+X \quad \text { with } X \in \mathfrak{u}^{\beta}
$$

is semisimple (ie diagonalizable). 
Remark 5.3 The above proposition is stated just for nilpotent groups since nilsolitons are precisely the critical points of $F=\|m\|^{2}$ (Theorem 2.2) and the automorphism group is the stabilizer of the $\operatorname{GL}(n, \mathbb{R})$ action. More generally, the above is true for solvable groups when $\mu$ is a critical point of $F=\|m\|^{2}$ (ie a distinguished metric) and $\alpha=m(\mu)$.

Before proving the proposition and lemma, we recall the following Morse theoretic stratification coming from $F=\|m\|^{2}$. The following are real analogues of classic results from Geometric Invariant Theory (over $\mathbb{C}$ ). Denote the critical set of $F=\|m\|^{2}$ by $\mathfrak{C}$.

Theorem 5.4 [20; 22] There exists a finite subset $B \subset \mathfrak{g l}(n, \mathbb{R})$ of diagonal elements with rational entries, and for each $\beta \in B$ a $\operatorname{GL}(n, \mathbb{R})$-invariant smooth submanifold $S_{\beta} \subset V$ (a stratum), such that $V \backslash\{0\}=\bigsqcup_{\beta \in B} S_{\beta}$.

This stratification satisfies $\bar{S}_{\beta}-S_{\beta}=\bigsqcup_{\left\|\beta^{\prime}\right\|>\|\beta\|} S_{\beta^{\prime}}$, the critical set is a union $\mathfrak{C}=$ $\bigsqcup_{\beta \in B} C_{\beta}$ where $C_{\beta} \subset S_{\beta}$ are the critical points with critical value $\|\beta\|^{2}$, and the $\beta$-stratum is $S_{\beta}=\left\{p \in V \mid \varphi_{\infty}(p) \in C_{\beta}\right\}$.

We use this stratification to prove the proposition above. Given symmetric $\alpha \in \mathfrak{g l}(n, \mathbb{R})$, consider the subgroup $H_{\alpha} \subset \mathrm{GL}(n, \mathbb{R})$ with Lie algebra $\mathfrak{h}_{\alpha}=\left\{X \in \mathfrak{g}_{\alpha} \mid \operatorname{tr}(X \alpha)=0\right\}$. This subgroup is self-adjoint, and algebraic when the eigenvalues of $\alpha$ are rational. In the following proposition, we maintain the notation from [20].

Definition 5.5 A point $v \in V$ is called $H_{\alpha}$-stable if $0 \notin \overline{H_{\alpha} \cdot v}$.

Proposition 5.6 (Lauret) Given $\beta \in B$, there exist subsets $Z_{\beta}$ and $Y_{\beta}$ with the following properties:

(1) $Y_{\beta}$ is $P_{\beta}$-invariant, $Y_{\beta}^{\text {ss }}=Y_{\beta} \cap S_{\beta}$ consists of $H_{\beta}$-semistable points and $S_{\beta}=O(n) Y_{\beta}^{\mathrm{ss}}$.

(2) For $y \in Y_{\beta}$, $\left\{g \in \mathrm{GL}(n, \mathbb{R}) \mid g \cdot y \in Y_{\beta}\right\}=P_{\beta}$.

(3) $Z_{\beta}=\left\{v \in Y_{\beta} \mid \pi(\beta) v=\|\beta\|^{2} v\right\}, Z_{\beta}$ is $G_{\beta}$-invariant, $S_{\beta} \cap Z_{\beta}=Z_{\beta}^{\text {ss }}$ (the $H_{\beta}$-semistable points of $\left.Z_{\beta}\right)$ and $S_{\beta}=\operatorname{GL}(n, \mathbb{R}) Z_{\beta}^{\text {ss }}=O(n) P_{\beta} Z_{\beta}^{\text {ss }}$.

(4) The $H_{\beta}$ orbits intersecting $Z_{\beta} \cap \mathfrak{C}_{\beta}$ are all closed.

Remark Part (2) above does not appear in [20]. However, one can show this immediately just as in the complex case (cf [16, Lemma 13.4]).

We are now in a position to prove Proposition 5.1 and Lemma 5.2. 
Proof of Proposition 5.1 This result follows quickly from Proposition 5.6. Let $\mu \in S_{\beta}$ be the nilsoliton of interest, where $S_{\beta}$ is the stratum defined above. By considering $O(n)$ translates of $\mu$, we may assume that $\mu \in Z_{\beta}$.

Let $g \in \operatorname{Aut}(\mu)$. Since $g \cdot \mu=\mu \in Z_{\beta} \subset Y_{\beta}, g \in P_{\beta}$ by Part (2) of Proposition 5.6, and we may write $g=g_{1} g_{2}$ where $g_{1} \in G_{\beta}$ and $g_{2} \in U_{\beta}$. Observe that $\exp (t \beta) g \exp (-t \beta)$ also stabilizes $\mu$ and letting $t \rightarrow-\infty$ we see that $g_{2} \in \operatorname{Aut}(\mu)$ and hence $g_{1} \in \operatorname{Aut}(\mu)$. This shows $\operatorname{Aut}(\mu)=G^{\beta} U^{\beta}$.

Given $g \in G^{\beta}$, write $g=k \exp (X)$ where $k \in K_{\beta}$ and $X \in \mathfrak{p}_{\beta}$; this is possible as $G_{\beta}$ is stable under the transpose. Observe, $\|m(\mu)\|=\|m(g \cdot \mu)\|=\|m(\exp (X) \cdot \mu)\|$ and by [27, Lemma 7.2] we see that $\exp (X) \cdot \mu=\mu$. Thus $\exp (X), k \in \operatorname{Aut}(\mu)$ and the theorem is proved.

Proof of Lemma 5.2 As $\mu$ is a nilsoliton with $\alpha=$ Ric, the automorphism group decomposes as $\operatorname{Aut}(\mu)=K^{\alpha} P^{\alpha} U^{\alpha}$. And since $\operatorname{Der}(\mu)=$ Lie $\operatorname{Aut}(\mu)$, we have $\operatorname{Der}(\mu)=\mathfrak{k}^{\alpha} \oplus \mathfrak{p}^{\alpha} \oplus \mathfrak{u}^{\alpha}$, where $\mathfrak{k}^{\alpha}=$ Lie $K^{\alpha}, P^{\alpha}=\exp \left(\mathfrak{p}^{\alpha}\right)$, and $\mathfrak{u}^{\alpha}=$ Lie $U^{\alpha}$. Now, if $\beta$ is the Einstein derivation (ie $\operatorname{Ric}=c \mathrm{Id}+\beta$ ), then clearly $K^{\alpha}=K^{\beta}$, etc.

The proof of the second statement is analogous to showing that any upper triangular matrix with nonzero distinct entries on the diagonal can be diagonalized. One carries out similar computations in this case (as the entries of $\beta$ are all positive and $\mathfrak{u}^{\beta}$ has an appropriate block structure) to show that $\beta+X$ can be conjugated to $\beta$ via $U_{\beta}$.

\section{Algorithm to determine Einstein nilradical}

In this section, we demonstrate how the existence of a nilsoliton can be read off from local data. In our setting, the phrase "local data" refers to invariants of the underlying Lie algebra and infinitesimal deformations of left-invariant metrics. Within the class of left-invariant metrics, an infinitesimal deformation of a left-invariant metric can be viewed as a perturbation of the inner product on the underlying Lie algebra.

Let $N$ be a nilpotent Lie group of interest with Lie algebra $\mathfrak{n}$.

Theorem 6.1 The existence of a nilsoliton metric on $N$ can be determined by analyzing algebraic invariants of $\mathfrak{n}$ and infinitesimal deformations of any initial left-invariant metric on $N$.

The existence of a nilsoliton being intrinsic to the Lie algebra was first shown by Nikolayevsky [31]. There it was shown that the existence of such a metric is equivalent to an orbit of a particular reductive group being closed in the space of Lie brackets (see 
Theorem 6.5). However, it was not shown that this could be determined by measuring local data. We build on this result and use a Hilbert-Mumford criterion to achieve the theorem above.

Before Nikolayevsky's result, it was shown by Lauret [17] that the existence of such a metric is equivalent to the full $\operatorname{GL}(n, \mathbb{R})$-orbit in the space of Lie brackets being so-called distinguished. However, it was not known before the present work that this condition can be determined locally.

\section{Algorithm to determine if $N$ is an Einstein nilradical}

Step 1 Find any nontrivial solution $\phi \in \operatorname{Der}(\mathfrak{n})$ to

$$
\operatorname{tr}(\phi \psi)=\operatorname{tr}(\psi) \quad \text { for all } \psi \in \operatorname{Der}(\mathfrak{n}) .
$$

If the solution is $\phi$ is not semisimple (ie diagonalizable) with (positive) real eigenvalues then stop, $\mathfrak{n}$ is not an Einstein nilradical.

If $\phi$ is semisimple with (positive) real eigenvalues, then continue. (Remark: positivity of the eigenvalues will be automatic if the following steps are valid.)

Step 2 Given $\phi$ above, define the subalgebra $g_{\phi}=\mathfrak{z}(\phi) \cap \operatorname{Ker}(T) \cap \mathfrak{s l}(n, \mathbb{R})$ of $\mathfrak{s l}(n, \mathbb{R})$, where $T$ is the linear function $T(A)=\operatorname{tr}(A \phi)$ and $\mathfrak{z}(\phi)$ is the centralizer of $\phi$ in $\mathfrak{g l}(n, \mathbb{R})$. Consider the subalgebra $\mathfrak{h}_{\phi}:=\mathfrak{g}_{\phi} \cap \operatorname{Der}(\mathfrak{n})$.

If $\mathfrak{h}_{\phi}$ is not reductive, then stop, $\mathfrak{n}$ is not an Einstein nilradical.

If $\mathfrak{h}_{\phi}$ is reductive then continue.

To determine if this algebra is reductive: (1) compute its radical, then (2) compute the set of nilpotent elements of this radical. The algebra is reductive if and only if the set of such nilpotent elements (in the radical) is trivial.

Step 3 Consider the subalgebra $i_{\phi}=\left\{X \in \mathfrak{z}_{\mathfrak{g}_{\phi}}\left(\mathfrak{h}_{\phi}\right) \mid \operatorname{tr}(X Y)=0\right.$ for all $\left.Y \in \mathfrak{z}\left(\mathfrak{h}_{\phi}\right)\right\}$, where $\mathfrak{z} a(b)$ denotes the centralizer of $b$ in $a$ and $\mathfrak{z}(b)$ is the center of $b$. Let $\mathcal{D}$ denote the matrices of $\mathfrak{g l}(n, \mathbb{R})$ which are diagonalizable over $\mathbb{R} ;$ ie, $\mathcal{D}=\bigcup_{g \in \mathrm{GL}(n, \mathbb{R})} g \mathfrak{t} g^{-1}$, where $\mathfrak{t}=$ diagonal matrices of $\mathfrak{g l}(n, \mathbb{R})$. Let $\mathfrak{n}=\mathfrak{n}_{\mu}$ corresponding to some point $\mu \in V=\wedge^{2}\left(\mathbb{R}^{n}\right)^{*} \otimes \mathbb{R}^{n}$ (see Section 2). For $X \in i_{\phi} \cap \mathcal{D}$, write $\mu=\sum a_{i} \mu_{i}$, where $\left\{\mu_{i}\right\}$ is an eigenbasis of $V$ relative to $X$, ie, $X \cdot \mu=\sum \lambda_{i} a_{i} \mu_{i}$.

If there is some $X \in i_{\phi} \cap \mathcal{D}$ such that $\lambda_{i} \geq 0$ whenever $a_{i} \neq 0$, then $\mathfrak{n}$ is not an Einstein nilradical.

If for every $X$ above there exists $i$ with $\lambda_{i}<0$ and $a_{i} \neq 0$, then $\mathfrak{n}$ is an Einstein nilradical. 
Remark 6.2 In Step 3 we have the following:

(1) The identification of $\mathfrak{n}$ with $\mu \in V$ is made by picking a basis of the vector space. This is tantamount to prescribing $\mathfrak{n}$ with an orthonormal basis, and hence, endowing $N$ with a choice of left-invariant metric.

(2) The $X \cdot \mu$, with $X \in \mathfrak{g l}(n, \mathbb{R})$, precisely represent infinitesimal deformations of the above choice of left-invariant metric.

(3) The algebra $\mathfrak{h}_{\phi}$ is reductive (once getting to Step 3). If the inner product from $\mathfrak{n}_{\mu}$ makes $\mathfrak{h}_{\phi}$ stable under the metric adjoint (and there will always be such a $\mu$ with this property), then Step 3 may be replaced by the following.

Step $3^{\prime}$ Assuming $\mathfrak{h}_{\phi}$ is self-adjoint relative to the inner product on $\mathfrak{n}_{\mu}$, we may reduce the collection of $X$ considered in Step 3 to those $X \in \mathfrak{i}_{\phi} \cap \operatorname{symm}(n)$, where $\operatorname{symm}(n)$ denotes the symmetric $n \times n$ matrices.

Remark 6.3 The verification of Steps 1 and 2 above can done by a computer. It is not immediately clear to the author if Step 3 can be adapted to be implemented by a computer.

\section{Proof of the algorithm above}

We begin by recalling a theorem Nikolayevsky, on which our work is built. Take $\mu \in \mathcal{V}$, with corresponding Lie algebra $\mathfrak{s} \mu$.

Definition 6.4 A derivation $\phi$ of a Lie algebra $\mathfrak{s}_{\mu}$ is called pre-Einstein if it is semisimple, with all eigenvalues real, and satisfies

$$
\operatorname{tr}(\phi \psi)=\operatorname{tr} \psi \quad \text { for any } \psi \in \operatorname{Der}(\mu)
$$

While these derivations exist for all Lie algebras, and are essentially unique, they are of particular interest when $\mu$ is nilpotent and admits a nilsoliton metric. Assume $\mu$ is said nilsoliton and let Ric $=c \operatorname{Id}+D$ be the Ricci tensor, then $\phi=\frac{D}{-c}$ is a preEinstein derivation, conversely, one can obtain $D$ from $\phi$. Remarkably, determining the pre-Einstein derivation almost completely determines when a nilpotent Lie algebra admits a nilsoliton metric. This derivation first appeared in [31].

Let $\mathfrak{n}_{\mu}$ be a nilpotent Lie algebra with a choice of pre-Einstein derivation $\phi$. Associated to $\phi$ we have the subalgebra $\mathfrak{g}_{\phi}=\mathfrak{z}(\phi) \cap \operatorname{ker}(T) \cap \mathfrak{s l}(n, \mathbb{R})$, where $\mathfrak{z}(\phi)$ is the centralizer of $\phi$ and $T(A)=\operatorname{tr}(A \phi)$. Let $G_{\phi} \subset \operatorname{SL}(n, \mathbb{R})$ be the Lie group with algebra $\mathfrak{g}_{\phi}$, this is an algebraic group. 
Theorem 6.5 [31] For a nilpotent Lie algebra $\mathfrak{n}_{\mu}$ with a pre-Einstein derivation $\phi$, the following conditions are equivalent:

(1) $\mathfrak{n}_{\mu}$ is an Einstein nilradical.

(2) The orbit $G_{\phi} \cdot \mu \subset V$ is closed.

We are now in a position to prove the algorithm.

Step 1 If $\mathfrak{n}_{\mu}$ is an Einstein nilradical, then any nontrivial solution to Equation (6-1) will automatically be a pre-Einstein derivation, ie it is automatically semisimple with real, positive eigenvalues.

Proof The proof amounts to analyzing Nikolayevsky's proofs and combining those details with Lemma 5.2. For the sake of completeness, we present Nikolayevsky's proof of existence and uniqueness (up to conjugation in Aut) of the pre-Einstein derivation.

First we find one pre-Einstein derivation. Let $\mathfrak{s}_{\mu}$ be a Lie algebra and denote by $\operatorname{Der}(\mu)$ its algebra of derivations; this is an algebraic Lie algebra (meaning it is the Lie algebra of an algebraic group). Consider a Levi decomposition $\operatorname{Der}(\mu)=\mathfrak{s} \oplus \mathfrak{t} \oplus \mathfrak{n}$ where $\mathfrak{t} \oplus \mathfrak{n}$ is the radical of $\operatorname{Der}(\mu), \mathfrak{s}$ is semisimple, and $\mathfrak{n}$ is the set of nilpotent elements (the nilradical) of $\mathfrak{t} \oplus \mathfrak{n}, \mathfrak{t}$ is a torus (abelian subalgebra with semisimple elements), and $[\mathfrak{t}, \mathfrak{s}]=0$.

Recall, for $\psi \in \mathfrak{g l}(n, \mathbb{R})$ a semisimple endomorphism, there exist semisimple endomorphisms $\psi^{\mathbb{R}}$ and $\psi^{i \mathbb{R}}$ (the real and imaginary parts) which have real, resp. purely imaginary, eigenvalues such that $\psi=\psi^{\mathbb{R}}+\psi^{i \mathbb{R}}$ and all three endomorphisms commute. Moreover, the subspaces $\mathfrak{t}_{c}=\left\{\psi^{i \mathbb{R}} \mid \psi \in \mathfrak{t}\right\}$ and $\mathfrak{t}_{s}=\left\{\psi^{\mathbb{R}} \mid \phi \in \mathfrak{t}\right\}$ are the compact and the fully R-reducible tori (the elements of $\mathfrak{t}_{s}$ are simultaneously diagonalizable) with $\mathfrak{t}_{s} \oplus \mathfrak{t}_{c}=\mathfrak{t}$.

We will find a pre-Einstein derivation contained in $\mathfrak{t}_{s}$. Consider the quadratic form $b$ on $\operatorname{Der}(\mu)$ defined by $b\left(\psi_{1}, \psi_{2}\right)=\operatorname{tr}\left(\psi_{1} \psi_{2}\right)$. It is a general fact that $\mathfrak{n}$ is in the kernel of this quadratic form, hence

$$
b(\mathfrak{t}, \psi)=0=\operatorname{tr}(\psi) \quad \text { for any } \psi \in \mathfrak{n} .
$$

Using the ad-invariance of $b\left(\right.$ ie, $\left.b\left(\psi_{1},\left[\psi_{2}, \psi_{3}\right]\right)=b\left(\left[\psi_{3}, \psi_{1}\right], \psi_{2}\right)\right)$ and that $\mathfrak{s}=[\mathfrak{s}, \mathfrak{s}]$ is semisimple, we see that

$$
b(\mathfrak{t}, \psi)=0=\operatorname{tr}(\psi) \quad \text { for any } \psi \in \mathfrak{s} .
$$

Thus it suffices to solve Equation (6-1) with $\phi, \psi \in \mathfrak{t}$. Additionally, observe that

$$
b\left(\mathfrak{t}_{s}, \psi\right)=0=\operatorname{tr}(\psi) \text { for any } \psi \in \mathfrak{t}_{c} .
$$


Lastly, as the quadratic form $b$ restricted to $\mathfrak{t}_{s}$ is positive definite, the existence (and uniqueness in $\mathfrak{t}$ ) follows.

To obtain the uniqueness of the pre-Einstein derivation up to conjugation in Aut, Nikolaevsky exploits a theorem of Mostow [26, Theorem 4.1] which says that all fully reducible subalgebras of $\operatorname{Der}(\mu)$ are conjugate via an inner automorphism of $\operatorname{Der}(\mu)$. Lastly, as the center of a reducible algebra is uniquely defined, we have the desired result.

Now we analyze this proof to study all solutions to Equation (6-1). Let $A \in \mathfrak{s} \oplus \mathfrak{t}$ be a solution to $\operatorname{tr}(A \psi)=0$ for all $\psi \in \operatorname{Der}(\mu)$. We will show that $A=0$. To see this, first assume that our Lie algebra $\mathfrak{n}_{\mu}$ is endowed with an inner product so that $\mathfrak{s} \oplus \mathfrak{t}$ is stable under the transpose operation. This is always possible; when $\mathfrak{n}_{\mu}$ is an Einstein nilradical such a metric is explicitly given in Proposition 5.1. Using this inner product, $\psi=A^{t} \in \operatorname{Der}(\mu)$ and $0=\operatorname{tr}(A \psi)=\operatorname{tr}\left(A A^{t}\right)$ implies $A=0$.

Let $\phi \in \mathfrak{t}$ be a pre-Einstein derivation of $\mathfrak{n}_{\mu}$, the above work shows that any solution to Equation (6-1) is of the form $\phi+X$ where $X \in \mathfrak{n}$ (the nilpotent part of the radical of $\operatorname{Der}(\mu))$. Applying Lemma 5.2 finishes the proof of this step.

Step 2 To prove this portion of the algorithm, we identify $\mathfrak{n}$ with $\mathfrak{n}_{\mu}$, for some $\mu \in V$. The algebra $\mathfrak{h}_{\phi}=\mathfrak{g}_{\phi} \cap \operatorname{Der}(\mathfrak{n})$ is precisely the stabilizer subalgebra of $\mathfrak{g}_{\phi}$ at $\mu$. As we have fixed a basis of our Lie algebra, we may view $\mathfrak{h}_{\phi} \subset \mathfrak{g l}(n, \mathbb{R})$.

In Theorem 6.5, it was shown that $\mathfrak{n}_{\mu}$ is an Einstein nilradical if and only if $G_{\phi} \cdot \mu$ is closed, where $G_{\phi}$ is the reductive algebraic Lie group with Lie algebra $\mathfrak{g}_{\phi}$. It is well-known that an orbit of such a group being closed implies the stabilizer subgroup is reductive; see [34]. Lastly, the stabilizer subgroup is reductive if and only if its Lie algebra $\mathfrak{h}_{\phi}$ is reductive.

Step 3 If the stabilizer of $G_{\phi}$ were finite, then we could apply the "Hilbert-Mumford criterion" to determine whether or not the orbit $G_{\phi} \cdot \mu$ is closed. This criterion uses only local data, and we reduce to this case with the following proposition.

Proposition 6.6 Consider $G_{\phi}$ as above and assume the subgroup $H_{\phi}=G_{\phi} \cap$ Aut is reductive. There exists a real algebraic reductive subgroup $I_{\phi}$ of $G_{\phi}$ satisfying the following.

(1) Lie $I_{\phi}=\mathfrak{i}_{\phi}=\left\{X \in \mathfrak{z}_{\mathfrak{g}_{\phi}}\left(\mathfrak{h}_{\phi}\right) \mid \operatorname{tr}(X Y)=0\right.$ for all $\left.Y \in \mathfrak{z}\left(\mathfrak{h}_{\phi}\right)\right\}$.

(2) The stabilizer of $I_{\phi}$ at $\mu$ is finite.

(3) $\mathfrak{n}_{\mu}$ is an Einstein nilradical if and only if $I_{\phi} \cdot \mu$ is closed. 
Before proving this proposition, we continue with the proof of the algorithm. As the stabilizer of $I_{\phi}$ at $\mu$ is finite, we may apply the "Hilbert-Mumford criterion" to determine when $I_{\phi} \cdot \mu$ is closed. This criterion was adapted to the real setting by Birkes [3] which states (in our setting):

$$
I_{\phi} \cdot \mu \text { is closed if and only if } \bigcup_{t \in \mathbb{R}} \exp (t X) \cdot \mu \text { is closed for all } X \in \mathcal{D} \cap \mathfrak{i}_{\phi} \text {. }
$$

Roughly speaking, this criterion says that closedness of the $I_{\phi}$-orbit is equivalent to closedness of the orbits of all algebraic reductive 1-parameter subgroups.

We finish by writing $\exp (t X) \cdot \mu=\operatorname{expt}(t X) \sum a_{i} \mu_{i}=\sum e^{t \lambda_{i}} a_{i} \mu_{i}$ where $\mu_{i}$ is the eigenvector of $X$ above. This set is not closed if and only if for all $i$ such that $a_{i} \neq 0$, either all $\lambda_{i} \geq 0$ or $\lambda_{i} \leq 0$. Observe that replacing $X$ with $-X$ changes the sign of the eigenvalues above and this step is proven.

Step 3' Reducing the Hilbert-Mumford criterion to this smaller set of symmetric elements of $\mathfrak{h}_{\phi}$ is the content of [34].

Proof of Proposition 6.6 The group $H_{\phi}$ is the (algebraic) Lie group with Lie algebra $\mathfrak{h}_{\phi}$. As $\mathfrak{h}_{\phi}$ and $\phi$ are reductive, there exists $g \in \operatorname{GL}(n, \mathbb{R})$ such that $g \mathfrak{h}_{\phi} g^{-1}$ and $g \phi g^{-1}$ are simultaneously self-adjoint; see [25]. Observe that

$$
\begin{aligned}
& g \operatorname{Der}(\mu) g^{-1}=\operatorname{Der}(g \cdot \mu), \\
& g \phi g^{-1} \text { is a pre-Einstein derivation of } g \cdot \mu, \\
& \mathfrak{g}_{g \phi g^{-1}}=g \mathfrak{g}_{\phi} g^{-1}, \\
& \mathfrak{h}_{g \phi g^{-1}}=g \mathfrak{h}_{\phi} g^{-1}, \\
& \mathfrak{z}_{\mathfrak{g}_{g \phi g^{-1}}}\left(\mathfrak{h}_{g \phi g^{-1}}\right)=g_{\mathfrak{z}_{\mathfrak{g}_{\phi}}}\left(\mathfrak{h}_{\phi}\right) g^{-1}, \\
& \mathfrak{i}_{g \phi g^{-1}}=g \mathfrak{i}_{\phi} g^{-1} .
\end{aligned}
$$

Moreover, $I_{g \phi g^{-1}} \cdot(g \cdot \mu)=g I_{\phi} g^{-1} g \mu=g\left(I_{\phi} \cdot \mu\right)$ is closed if and only if $I_{\phi} \cdot \mu$ is closed. As such, we may reduce to the case that $\mathfrak{h}_{\phi}$ and $\phi$ are self-adjoint.

Since $\phi$ is self-adjoint, we immediately have that $\mathfrak{g}_{\phi}$ and $\mathfrak{z}_{\mathfrak{g}_{\phi}}\left(\mathfrak{h}_{\phi}\right)$ are self-adjoint. Observe that the stabilizer subalgebra of $\mathfrak{i}_{\phi}$ at $\mu$ is trivial since it is contained in the stabilizer subalgebra $\left(\mathfrak{g}_{\phi}\right)_{\mu}=\mathfrak{h}_{\phi}$ and $\mathfrak{i}_{\phi}$ is orthogonal to $\mathfrak{h}_{\phi}$ under the inner product $\langle A, B\rangle=\operatorname{tr}\left(A B^{t}\right)$. Once $I_{\phi}$ is shown to be algebraic, we immediately have that the stabilizer of $I_{\phi}$ at $\mu$ is finite, as it is discrete and algebraic. 
Algebraicity of $I_{\phi} \quad$ We include a proof of this fact as such details are often not readily available in the literature (for real algebraic groups) and, to apply the tools from geometric invariant theory, the group must be algebraic.

An element $X \in \mathfrak{g l}(n, \mathbb{R})$ will be called algebraic if it is tangent to a real algebraic 1 -parameter subgroup of $\operatorname{GL}(n, \mathbb{R})$. More generally, a Lie subalgebra will be called algebraic if it is tangent to an algebraic subgroup of $\operatorname{GL}(n, \mathbb{R})$. Given $\alpha$ which is symmetric or skew-symmetric, we define the subalgebra

$$
\mathfrak{g}_{\alpha} \ominus \alpha=\left\{X \in \mathfrak{g}_{\alpha} \mid \operatorname{tr}\left(X \alpha^{t}\right)=0\right\}
$$

where $\mathfrak{g}_{\alpha}=\{X \in \mathfrak{g} \mid[X, \alpha]=0\}$. Since $\alpha^{t}= \pm \alpha$, it follows that $\mathfrak{g}_{\alpha} \ominus \alpha$ is a self-adjoint ideal of $\mathfrak{g}_{\alpha}$.

Lemma 6.7 Given $\alpha$ which is algebraic and either symmetric or skew-symmetric, the subalgebra $\mathfrak{g}_{\alpha} \ominus \alpha$ is an algebraic Lie subalgebra.

Using this lemma, we show that $I_{\phi}$ is algebraic. Observe that the algebra $\mathfrak{z}\left(\mathfrak{h}_{\phi}\right)=$ $\mathfrak{z}_{\mathfrak{g}_{\phi}}\left(\mathfrak{h}_{\phi}\right) \cap \mathfrak{h}_{\phi}$ is algebraic and self-adjoint, and decompose $\mathfrak{z}\left(\mathfrak{h}_{\phi}\right)=\mathfrak{z}\left(\mathfrak{h}_{\phi}\right)_{\mathfrak{k}} \oplus \mathfrak{z}\left(\mathfrak{h}_{\phi}\right)_{\mathfrak{p}}$ into its Cartan decomposition (the skew-symmetric and symmetric components). The algebraic elements of $\mathfrak{z}\left(\mathfrak{h}_{\phi}\right)_{\mathfrak{k}}$ and $\mathfrak{z}\left(\mathfrak{h}_{\phi}\right)_{\mathfrak{p}}$ are dense in each subspace. Applying the above lemma to all of these algebraic elements, and using the fact that the intersection of algebraic groups is algebraic, we see that $I_{\phi}$ is algebraic.

Proof of Lemma 6.7 The cases of $\alpha$ symmetric and skew-symmetric must be handled separately.

Case $\alpha$ is symmetric. Every such $\alpha$ is conjugate via $O(n, \mathbb{R})$ to a diagonal matrix. As the bilinear form $\operatorname{tr}\left(X Y^{t}\right)$ on $\mathfrak{g l}(n, \mathbb{R})$ is $\operatorname{Ad} O(n, \mathbb{R})$ invariant and the conjugate of an algebraic group is algebraic, we may reduce to the case that $\alpha$ is diagonal. Also, we may reduce to the case $G=\operatorname{GL}(n, \mathbb{R})$ as the intersection of algebraic groups is algebraic.

Further more, we may assume (via conjugation by $O(n, \mathbb{R})$ ) that the eigenvalues are weakly increasing: $\alpha=\operatorname{diag}\left\{a_{1}, \ldots, a_{1}, \ldots, a_{k}, \ldots, a_{k}\right\}$. After rescaling, the eigenvalues $a_{i}$ are rational as $\alpha$ is algebraic. Now the subalgebra $\mathfrak{g}_{\alpha}$ consists of block diagonal matrices $\mathfrak{g l}\left(n_{1}, \mathbb{R}\right) \times \cdots \times \mathfrak{g l}\left(n_{k}, \mathbb{R}\right)$. This is clearly an algebraic Lie algebra whose Lie group $G_{\alpha}$ consists of the block matrices which are invertible.

The condition $X \in \mathfrak{g}_{\alpha}$ is now $\Sigma a_{i} X_{i}=0$ where $X=\operatorname{blockdiag}\left\{X_{1}, \ldots, X_{k}\right\}$. Write $g \in G_{\alpha}$ as a block diagonal matrix $g=\operatorname{blockdiag}\left\{g_{1}, \ldots, g_{k}\right\}$. Then the algebraic group with Lie algebra $\mathfrak{g}_{\alpha} \ominus \alpha$ is

$$
\left\{g \in G_{\alpha} \mid \prod \operatorname{det}\left(g_{i}\right)^{q a_{i}}=1\right\}
$$

where $q$ is the common integer such that $q a_{i} \in \mathbb{Z}$ for all $i=1, \ldots, k$. 
Case $\alpha$ is skew-symmetric. To prove the result in this case, we reduce to the above case and use complex algebraic groups. We will construct a complex algebraic group whose intersection with $\operatorname{GL}(n, \mathbb{R})$ is the desired Lie group. This Lie group will be algebraic as it is the intersection of algebraic groups. We refer the reader to Whitney [36] for an introduction to the relationship between real and complex varieties.

Observe that the above work for $\alpha$ symmetric could have been carried out over $\mathbb{C}$. Consider $\mathfrak{g}_{\alpha}^{\mathbb{C}}=\{X \in \mathfrak{g l}(n, \mathbb{C}) \mid[X, \alpha]=0\}$. As $\alpha$ is skew-symmetric, the eigenvalues of $i \alpha$ are real (and may be assumed to be rational, as above). Now we have $\mathfrak{g}_{\alpha}^{\mathbb{C}}=\mathfrak{g}_{i \alpha}^{\mathbb{C}}$ and $\mathfrak{g}_{\alpha}^{\mathbb{C}} \ominus \alpha=\mathfrak{g}_{i \alpha}^{\mathbb{C}} \ominus i \alpha$. By conjugating with $U(n) \subset \mathrm{GL}(n, \mathbb{C})$, we may assume $i \alpha$ is diagonal. Following the above work, but with complex groups instead of real, we have a complex algebraic group over $\mathfrak{g}_{\alpha}^{\mathbb{C}} \ominus \alpha=\mathfrak{g}_{i \alpha}^{\mathbb{C}} \ominus i \alpha$. Moreover, this group intersected with $\operatorname{GL}(n, \mathbb{R})$ is a real Lie group with the desired Lie algebra. Counting dimensions, we see that the real points of this complex algebraic group are Zariksi dense and hence this Lie group is real algebraic.

\section{Einstein nilradical vs closedness of $I_{\phi}$-orbit}

By Theorem 6.5, $\mathfrak{n}_{\mu}$ is an Einstein nilradical if and only if $G_{\phi} \cdot \mu$ is closed. However, by Lemma $4.5, G_{\phi} \cdot \mu$ is closed if and only if $Z_{G_{\phi}}\left(H_{\phi}\right)$ is closed. Now, by the construction of $I_{\phi}$, we have $Z_{G_{\phi}}\left(H_{\phi}\right)=I_{\phi} \cdot Z\left(H_{\phi}\right)$ and $I_{\phi} \cdot \mu=Z_{G_{\phi}}\left(H_{\phi}\right) \cdot \mu$. This proves the proposition and the proof of the algorithm is complete.

\section{Closed orbits for general representations}

The above work concerning the geometry of orbits holds in the more general framework of representations of reductive groups. We state this result without proof, as the proof is similar to the above work. Although probably known to experts, we do not know of this statement appearing in the literature before.

Theorem 6.8 Let $G$ be a real reductive algebraic group acting linearly and rationally on a vector space $V$. Determining whether an orbit $G \cdot v$ is closed in $V$ can be determined using only data from the induced representation of the Lie algebra $\mathfrak{g}$ at the point $v \in V$.

\section{Algorithm to determine if a solvable Lie group admits a left-invariant Einstein metric}

The existence of an Einstein metric on a solvable Lie group can be determined by measuring purely local data, as in the case of nilsolitons and nilpotent Lie groups; 
see Section 6. The following theorem builds on the works of Heber, Lauret, and Nikolayevsky. (A similar algorithm can be written to test for the existence of solvsoliton metrics.)

Theorem 7.1 The existence of an Einstein metric on a solvable Lie group $S$ can be determined by analyzing algebraic invariants of $\mathfrak{s}$ and infinitesimal deformations of any initial left-invariant metric on $S$.

As (nonabelian) solvable Lie groups are noncompact, any left-invariant Einstein metric on such a group must have scalar curvature less than or equal to zero by Bonnet-Myers theorem. Using the following result, we distinguish between zero and negative curvature by means of algebra.

Theorem 7.2 (Alekseevskiı̌-Kimel'fel'd [1], Dotti [5]) Let $S$ be a solvable Lie group with left-invariant Einstein metric.

(1) The scalar curvature is negative if and only if $S$ is nonunimodular.

(2) The scalar curvature is zero if and only if $S$ is unimodular.

In the case of Ricci flat, the metric is actually flat (ie, constant zero sectional curvature).

Remark In [1] it is actually shown that any homogeneous Ricci flat space must be flat.

This result is the first step in proving Theorem 7.1:

- If the underlying Lie algebra is unimodular, check to see if the group admits a flat metric.

- If the underlying Lie algebra is nonunimodular, check to see if the group admits a negative Einstein metric.

\section{Flat Einstein metrics}

Lie groups with flat left-invariant metrics are necessarily unimodular solvable and have been classified [24].

Theorem 7.3 (Milnor) A Riemannian Lie group $S$ is flat if and only if its Lie algebra $\mathfrak{s}$ (with inner product) splits as an orthogonal direct sum $\mathfrak{s}=\mathfrak{a} \oplus \mathfrak{n}$ where $\mathfrak{n}$ is an abelian ideal (the nilradical) and $\mathfrak{a}$ is an abelian Lie algebra such that ad $X$ is skew-symmetric for $X \in \mathfrak{a}$. Such $S$ is necessarily solvable. 
Using Milnor's classification, we prove Theorem 7.1 in the case that scalar curvature is zero. Note: this case does not require any infinitesimal deformations of metrics on $\mathfrak{n}$.

Consider the adjoint action $\operatorname{ad} \mathfrak{s} \subset \operatorname{Der}(\mathfrak{s})$ on $\mathfrak{s}$. Compute the nilradical $\mathfrak{n}$ of $\mathfrak{s}$, ie, the set of nilpotent elements. Compute any Levi decomposition ad $\mathfrak{s}=\mathfrak{T}+\mathfrak{N}$, where $\mathfrak{N}$ is the nilradical and $\mathfrak{T}$ is a reductive compliment. Let $\mathfrak{t} \subset \mathfrak{s}$ be any preimage of $\mathfrak{T}$ (ie, $\operatorname{ad} \mathfrak{t}=\mathfrak{T}$ ) such that $\operatorname{dim} \mathfrak{t}=\operatorname{dim} \mathfrak{T}$.

Lemma 7.4 A (unimodular) solvable Lie group $S$ admits a flat metric if and only if the nilradical $\mathfrak{n}$ is abelian, $\mathfrak{t}$ is an abelian subalgebra, and ad $T$ has only purely imaginary eigenvalues for $T \in \mathfrak{t}$.

Proving this lemma proves Theorem 7.1 in case of zero curvature as verifying the conditions on $\mathfrak{t}$ in the lemma amount to simply analyzing the adjoint representation of $\mathfrak{s}$.

Proof of Lemma 7.4 We prove necessity first. Assume $\mathfrak{s}$ admits a flat Einstein metric. Decompose $\mathfrak{s}=\mathfrak{a}+\mathfrak{n}$ where $\mathfrak{n}$ is the nilradical and $\mathfrak{a}$ is an abelian, reductive subalgebra such that ad $A$ has only purely imaginary eigenvalues for $A \in \mathfrak{a}$, as in Theorem 7.3.

Observe that $\operatorname{ad} \mathfrak{s}=\operatorname{ad} \mathfrak{a}+\operatorname{ad} \mathfrak{n}$ is a Levi-decomposition of ad $\mathfrak{s}$. Thus ad $\mathfrak{a}$ and $\mathfrak{T}=\operatorname{ad} \mathfrak{t}$ are equal up to conjugation by $\operatorname{Aut}(\mathfrak{s})$ as they are both maximal reductive subalgebras of ad $\mathfrak{s}$ (conjugacy of such subalgebras is the main result of [26]). As the relevant properties of $\mathfrak{a}$ do not change after applying an automorphism, we may assume $\operatorname{ad} \mathfrak{a}=\operatorname{ad} \mathfrak{t}$. Now, the elements of $\mathfrak{t}$ differ from the elements of $\mathfrak{a}$ by only elements of the center. Hence $\mathfrak{t}$ has precisely the same properties of $\mathfrak{a}$ and this direction of the lemma is proven.

We prove sufficiency. Picking a basis of $\mathfrak{n}$, we may identify it with $\mathbb{R}^{n}$. Via this identification, the abelian, reductive algebra $\mathfrak{t}$ is a subalgebra of $\mathfrak{g l}(n, \mathbb{R})$. It is wellknown that there exists an inner product on $\mathbb{R}^{n}$ such that $\mathfrak{t}$ is stable under the transpose operation; see [25]. As the eigenvalues of every element in $\mathfrak{t}$ are purely imaginary, we see that $\mathfrak{t}$ consists of skew-symmetric derivations. Now Milnor's theorem above applies.

\section{Negative Einstein metrics}

Here we prove Theorem 7.1 in the case that scalar curvature is negative. Let $S$ be the solvable group in question with Lie algebra $\mathfrak{s}$. Denote by $\mathfrak{n}$ the commutator subalgebra $[\mathfrak{s}, \mathfrak{s}]$ of $\mathfrak{s}$ (when $\mathfrak{s}$ admits an Einstein metric, this will be the full nilradical). We prove the theorem by producing an algorithm. 


\section{Step 1}

- If $\mathfrak{n}$ is not an Einstein nilradical, then stop, $S$ cannot admit a negative Einstein metric.

- If $\mathfrak{n}$ is an Einstein nilradical, then continue.

This step can be determined using the algorithm of Section 6 .

Step 2 Find any nontrivial solution $\phi$ to

$$
\operatorname{tr}(\phi \psi)=\operatorname{tr}(\psi) \quad \text { for all } \psi \in \operatorname{Der}(\mathfrak{n})
$$

within the set $\operatorname{ad} \mathfrak{s}=\{\operatorname{ad} X \mid X \in \mathfrak{s}\} \subset \operatorname{Der}(\mathfrak{n})$.

- If there is no nontrivial solution in this subset, or the solution is not semisimple with (positive) real eigenvalues, then stop; $S$ cannot admit a negative Einstein metric.

- If there is a nontrivial solution $\phi=\operatorname{ad} X_{\phi}$, and this solution is semisimple with (positive) real eigenvalues, then continue. Fix this choice of $X_{\phi}$.

This step can be verified using a computer for a given solvable Lie algebra of interest. As before, positivity of the eigenvalues will follow if the remaining steps are valid.

Step 3 Compute $\mathfrak{z}_{\mathfrak{s}}\left(X_{\phi}\right)=\left\{Y \in \mathfrak{s} \mid\left[Y, X_{\phi}\right]=0\right\}$.

- If $\mathfrak{z}_{\mathfrak{s}}\left(X_{\phi}\right)$ is not abelian or $\operatorname{dim} \mathfrak{z}_{\mathfrak{s}}\left(X_{\phi}\right)+\operatorname{dim} \mathfrak{n}<\operatorname{dim} \mathfrak{s}$, then stop, $S$ does not admit a negative Einstein metric.

- If $\mathfrak{z}_{\mathfrak{s}}\left(X_{\phi}\right)$ is abelian, and $\operatorname{dim} \mathfrak{z}_{\mathfrak{s}}\left(X_{\phi}\right)+\operatorname{dim} \mathfrak{n}=\operatorname{dim} \mathfrak{s}$, then continue.

Recall, $\mathfrak{z}_{\mathfrak{s}}\left(X_{\phi}\right)$ is automatically reductive as $X_{\phi}$ is reductive, and $\mathfrak{z}_{\mathfrak{s}}\left(X_{\phi}\right)$ being reductive abelian implies that no element is nilpotent. This step may be verified using a computer.

\section{Step 4}

- If some element of $\mathfrak{z}_{\mathfrak{s}}\left(X_{\phi}\right)$ has only purely imaginary eigenvalues, then stop; $S$ does not admit a negative Einstein metric.

- If no element of $\mathfrak{z}_{\mathfrak{s}}\left(X_{\phi}\right)$ has only purely imaginary eigenvalues, then $S$ admits a negative Einstein metric. 


\section{Proof of the algorithm above}

Step 1 This fact is well-known; see [20].

Step 2 This is a slight refinement of a result of Nikolayevsky [31, Theorem 1] and Lauret [21, Proposition 4.3]. The proof is the same as Step 1 of the algorithm in Section 6.

Step 3 This holds immediately by the structural results of Lauret [21, Theorem 4.8].

Step 4 This step gives algebraic form to the rigid structural results of Lauret [21, Theorem 4.8] for Einstein metrics on nonunimodular solvable Lie groups. We prove this below.

Denote $\mathfrak{z}_{\mathfrak{s}}\left(X_{\phi}\right)$ by $\mathfrak{a}$ and consider it as a subalgebra of $\mathfrak{g l}\left(\mathfrak{n}_{\mu}\right)$. Let $A$ be the connected subgroup of GL $\left(\mathfrak{n}_{\mu}\right)$ with Lie algebra $\mathfrak{a}$. Denote by $\bar{A}$ the Zariski closure of $A$ in $\operatorname{GL}\left(\mathfrak{n}_{\mu}\right)$ (ie, the smallest algebraic group containing $A$ ) and its Lie algebra by $\overline{\mathfrak{a}}$. As $\operatorname{Aut}\left(\mathfrak{n}_{\mu}\right)$ is an algebraic group, $\bar{A} \subset \operatorname{Aut}\left(\mathfrak{n}_{\mu}\right)$. Moreover, $\bar{A}$ is abelian and reductive. The fact that $\bar{A}$ is abelian follows immediately from being the closure of an abelian group. To see that this group is reductive, one can "diagonalize" $\mathfrak{a}$ to see that $\bar{A}$ is a subgroup of a torus (abelian, reductive) of $\operatorname{GL}\left(\mathfrak{n}_{\mu}\right)$ and hence has no nontrivial nilpotent elements.

It is a classical fact that there exists $g \in \operatorname{GL}(n, \mathbb{R})$ such that $g \overline{\mathfrak{a}} g^{-1}$ is self-adjoint since $\bar{A}$ is both algebraic and reductive; see [25]. We note that the eigenvalue conditions on $\mathfrak{a}$ do not change under conjugation. Now $\mathfrak{a}_{0}=g \mathfrak{a} g^{-1}$ is a reductive, abelian subalgebra of $\operatorname{Der}\left(g^{*} \mu\right)$ and

$$
\mathfrak{a} \ltimes \mathfrak{n}_{\mu} \simeq \mathfrak{a}_{0} \ltimes \mathfrak{n}_{g^{*} \mu}
$$

via the isomorphism which is the identity on $\mathfrak{a}$ and $g$ on $\mathfrak{n}_{\mu}$. The nilpotent Lie group $N_{g^{*} \mu}$ is an Einstein nilradical if and only if $N_{\mu}$ is so, as they are isomorphic.

We apply Lemma 4.5 to the subgroup $\bar{A}_{0}=g \bar{A} g^{-1} \subset \mathrm{GL}(n, \mathbb{R})$ with Lie algebra $\overline{\mathfrak{a}_{0}}=$ $g \bar{a} g^{-1}$. This group is self-adjoint as its Lie algebra is so. Let $\mu_{0}=g^{*} \mu$ and consider the limit $\mu_{\infty}$ of the bracket flow $\mu_{t}$. The Riemannian nilpotent Lie group $N_{\mu_{\infty}}$ is a nilsoliton and, by Theorem $2.7, \mu_{\infty}=g^{\prime *} \mu_{0}$ for some $g^{\prime} \in Z_{\mathrm{GL}(n, \mathbb{R})}\left(\bar{A}_{0}\right)$. As $g^{\prime}$ commutes with $\mathfrak{a}_{0}$ we see that the following solvable algebras are isomorphic:

$$
\mathfrak{a} \ltimes \mathfrak{N}_{\mu} \simeq \mathfrak{a}_{0} \ltimes \mathfrak{N}_{\mu_{0}} \simeq g^{\prime} \mathfrak{a}_{0} g^{\prime-1} \ltimes \mathfrak{N}_{g^{\prime *} \mu_{0}}=\mathfrak{a}_{0} \ltimes \mathfrak{N}_{g^{\prime *} \mu_{0}}=\mathfrak{a}_{0} \ltimes \mathfrak{N}_{\mu_{\infty}},
$$

but the last metric algebra satisfies all of Lauret's the criteria [21, Theorem 4.8] to be an Einstein metric Lie algebra. 


\section{References}

[1] D V Alekseevskiǐ, B N Kimel'fel'd, Structure of homogeneous Riemannian spaces with zero Ricci curvature, Funkcional. Anal. i PriloŽen. 9 (1975) 5-11 MR0402650 In Russian

[2] A L Besse, Einstein manifolds, Classics in Math., Springer, Berlin (2008) MR2371700 Reprint of the 1987 edition

[3] D Birkes, Orbits of linear algebraic groups, Ann. of Math. (2) 93 (1971) 459-475 MR0296077

[4] C Böhm, M Wang, W Ziller, A variational approach for compact homogeneous Einstein manifolds, Geom. Funct. Anal. 14 (2004) 681-733 MR2084976

[5] I Dotti Miatello, Ricci curvature of left invariant metrics on solvable unimodular Lie groups, Math. Z. 180 (1982) 257-263 MR661702

[6] P Eberlein, Riemannian 2-step nilmanifolds with prescribed Ricci tensor, from: "Geometric and probabilistic structures in dynamics", (K Burns, D Dolgopyat, Y Pesin, editors), Contemp. Math. 469, Amer. Math. Soc. (2008) 167-195 MR2478470

[7] P Eberlein, M Jablonski, Closed orbits of semisimple group actions and the real Hilbert-Mumford function, from: "New developments in Lie theory and geometry", (C S Gordon, J Tirao, J A Vargas, J A Wolf, editors), Contemp. Math. 491, Amer. Math. Soc. (2009) 283-321 MR2537062

[8] C S Gordon, E N Wilson, Isometry groups of Riemannian solvmanifolds, Trans. Amer. Math. Soc. 307 (1988) 245-269 MR936815

[9] J Heber, Noncompact homogeneous Einstein spaces, Invent. Math. 133 (1998) 279-352 MR1632782

[10] P Heinzner, G W Schwarz, H Stötzel, Stratifications with respect to actions of real reductive groups, Compos. Math. 144 (2008) 163-185 MR2388560

[11] M Jablonski, Distinguished orbits of reductive groups, to appear in Rocky Mountain J. Math. arXiv:0806.3721

[12] M Jablonski, Detecting orbits along subvarieties via the moment map, Münster J. Math. 3 (2010) 67-88

[13] M Jablonski, Moduli of Einstein and non-Einstein nilradicals, Geom. Dedicata 152 (2011) 63-84

[14] G R Jensen, Homogeneous Einstein spaces of dimension four, J. Differential Geometry 3 (1969) 309-349 MR0261487

[15] G Kempf, L Ness, The length of vectors in representation spaces, from: "Algebraic geometry (Proc. Summer Meeting, Univ. Copenhagen, 1978)", (K Lønsted, editor), Lecture Notes in Math. 732, Springer, Berlin (1979) 233-243 MR555701 
[16] F C Kirwan, Cohomology of quotients in symplectic and algebraic geometry, Math. Notes 31, Princeton Univ. Press (1984) MR766741

[17] J Lauret, Ricci soliton homogeneous nilmanifolds, Math. Ann. 319 (2001) 715-733 MR1825405

[18] J Lauret, On the moment map for the variety of Lie algebras, J. Funct. Anal. 202 (2003) 392-423 MR1990531

[19] J Lauret, Einstein solvmanifolds and nilsolitons, from: "New developments in Lie theory and geometry", (C S Gordon, J Tirao, J A Vargas, J A Wolf, editors), Contemp. Math. 491, Amer. Math. Soc. (2009) 1-35 MR2537049

[20] J Lauret, Einstein solvmanifolds are standard, Ann. of Math. (2) 172 (2010) 18591877 MR2726101

[21] J Lauret, Ricci soliton solvmanifolds, J. Reine Angew. Math. 650 (2011) 1-21

[22] J Lauret, C Will, Einstein solvmanifolds: existence and non-existence questions, Math. Ann. 350 (2011) 199-225

[23] A Marian, On the real moment map, Math. Res. Lett. 8 (2001) 779-788 MR1879820

[24] J Milnor, Curvatures of left invariant metrics on Lie groups, Advances in Math. 21 (1976) 293-329 MR0425012

[25] G D Mostow, Self-adjoint groups, Ann. of Math. (2) 62 (1955) 44-55 MR0069830

[26] G D Mostow, Fully reducible subgroups of algebraic groups, Amer. J. Math. 78 (1956) 200-221 MR0092928

[27] L Ness, A stratification of the null cone via the moment map, Amer. J. Math. 106 (1984) 1281-1329 MR765581 With an appendix by D Mumford

[28] Y Nikolayevsky, Einstein solvmanifolds attached to two-step nilradicals arXiv: $0805.0646 \mathrm{v} 1$

[29] Y Nikolayevsky, Einstein solvmanifolds with a simple Einstein derivation, Geom. Dedicata 135 (2008) 87-102 MR2413331

[30] Y Nikolayevsky, Einstein solvmanifolds with free nilradical, Ann. Global Anal. Geom. 33 (2008) 71-87 MR2369187

[31] Y Nikolayevsky, Einstein solvmanifolds and the pre-Einstein derivation, Trans. Amer. Math. Soc. 363 (2011) 3935-3958

[32] Y G Nikonorov, Noncompact homogeneous Einstein 5-manifolds, Geom. Dedicata 113 (2005) 107-143 MR2171301

[33] T L Payne, The existence of soliton metrics for nilpotent Lie groups, Geom. Dedicata 145 (2010) 71-88 MR2600946

[34] R W Richardson, P J Slodowy, Minimum vectors for real reductive algebraic groups, J. London Math. Soc. (2) 42 (1990) 409-429 MR1087217 
[35] R Sjamaar, Convexity properties of the moment mapping re-examined, Adv. Math. 138 (1998) 46-91 MR1645052

[36] H Whitney, Elementary structure of real algebraic varieties, Ann. of Math. (2) 66 (1957) 545-556 MR0095844

[37] C Will, Rank-one Einstein solvmanifolds of dimension 7, Differential Geom. Appl. 19 (2003) 307-318 MR2013098

[38] C Will, A curve of nilpotent Lie algebras which are not Einstein nilradicals, Monatsh. Math. 159 (2010) 425-437 MR2600907

Department of Mathematics, University of Oklahoma

Norman OK 73019-0315, USA

mjablonski@math.ou.edu

http://www.math.ou.edu/ mjablonski/

Proposed: Dmitri Burago

Seconded: Frances Kirwan, Simon Donaldson

Received: 26 September 2010

Revised: 13 January 2011 